Abanico Veterinario. Enero-Diciembre 2021; 11:1-16. http://dx.doi.org/10.21929/abavet2021.17 Artículo Original. Recibido: 25/09/2020. Aceptado: 24/03/2021. Publicado: 10/04/2021. Clave: e2020-85.

\title{
Perfiles antropométricos y de lípidos séricos en hombres con sobrepeso que consumieron carne de cerdo alimentado con harina de aguacate
}

\author{
Anthropometric and serum lipid profiles in overweight men who consumed pork fed \\ avocado flour
}

\section{González-Jiménez Cecilia ${ }^{11 \mathrm{D}}$, Lemus-Flores Clemente ${ }^{2 \mathrm{ID}}$, Becerra-Verdín Eduardo $^{21 \mathrm{D}}$, Bugarín-Prado Job ${ }^{21 \mathrm{D}}$, Mejía-Martínez Karina ${ }^{21 \mathrm{D} *}$}

${ }^{1}$ Universidad Autónoma de Nayarit. México. Estudiante de doctorado del programa del posgrado en ciencias biológicas y agropecuarias. Nayarit, México. ${ }^{2}$ Universidad Autónoma de Nayarit. México. Área de Ciencias Biológico Agropecuarias y Pesqueras. *Autor responsable y de correspondencia: Mejía-Martínez Karina. Km. 9 Carretera Tepic-Compostela, 63780 Xalisco, Nayarit, México. anacecyglez@gmail.com, clemus23@gmail.com, eduardo.becerra@uan.edu.mx, job.bugarin@uan.edu.mx, karinamej13@gmail.com.

\section{RESUMEN}

La alimentación humana puede regularse para generar efectos positivos en la salud cardiovascular. El objetivo fue evaluar el efecto del consumo de carne de cerdo alimentado con harina de aguacate al $10 \%$, sobre perfiles antropométricos y lípidos en suero de humanos con sobrepeso en dos periodos de tiempo. Se conformaron dos grupos de 10 participantes cada uno, masculinos de 30 a 60 años con IMC >27 kg/m². Consumieron diario $100 \mathrm{~g}$ de lomo de cerdo, el grupo A por cuatro semanas y grupo B por seis semanas. Se realizaron análisis con prueba de T pareada $(p<0.05)$. Grupo A disminuyó grasa corporal $(-4.04)$, IMC (-0.92), triglicéridos (-51.6), VLDL (-10.32) y aumentó LDL (48.98). Grupo B disminuyó grasa corporal (1.28), IMC $(-1,26)$, grasa visceral $(-0.4)$, cintura $(-4.4)$, glucosa $(-18.5)$. Usando prueba de $X^{2}(p<0.05)$, se compararon las proporciones de niveles bajos, normales o altos, después del consumo. En el grupo A se normalizaron los valores glucosa y triglicéridos, se incrementó la frecuencia del nivel alto para colesterol y LDL, con disminución de frecuencia al nivel bajo para VLDL. Grupo B, se normalizaron los valores para glucosa, colesterol, triglicéridos, TGO, HDL y LDL; para VLDL tuvieron mayor porcentaje de participantes a nivel bajo. El consumo de lomo de cerdos que fueron alimentados con harina de aguacate al $10 \%$, contribuye a la inducción de efectos saludables en consumidores con sobrepeso.

Palabras clave: Harina de aguacate, perfil de lípidos séricos, carne de cerdo, sobrepeso, dieta.

\begin{abstract}
Human diet can be regulated to generate positive effects on cardiovascular health. The objective was to evaluate the effect of pork consumption fed with $10 \%$ avocado flour on anthropometric profiles and serum lipids in overweight humans over two time periods. Two groups of 10 participants each, males aged 30 to 60 years with $\mathrm{BMl}>27 \mathrm{~kg} / \mathrm{m}^{2}$, were formed. $100 \mathrm{~g}$ of pork loin were consumed daily, group A for four weeks and group B for six weeks. Paired t-test analysis was performed $(p<0.05)$. Group A decreased body fat (-4.04), BMI (-0.92), triglycerides (-51.6), VLDL (-10.32) and increased LDL (48.98). Group B decreased body fat (-1.28), BMI (-1.26), visceral fat $(-0.4)$, waist $(-4.4)$, glucose $(-18.5)$. Using the $X^{2}$ test $(p<0.05)$, the proportions of low, normal or high levels were compared after consumption. In group A, glucose and triglyceride values were normalized, the frequency of the high level for cholesterol and LDL increased, with a decrease in the frequency of low level for VLDL. Group B, the values for glucose, cholesterol, triglycerides, TGO, HDL and LDL were normalized; for VLDL there was a higher percentage of participants at the low level. The consumption of pork loins from pigs fed $10 \%$ avocado meal contributes to the induction of health effects in overweight consumers.
\end{abstract}

Keywords: Avocado flour, serum lipid profile, pork, overweight, diet. 


\section{INTRODUCCIÓN}

La alimentación humana puede regularse para generar efectos positivos en la salud cardiovascular (Berciano y Ordovás, 2014). Las enfermedades cardiovasculares son la principal causa de muerte en todo el mundo, por lo que se sigue buscando las recomendaciones nutricionales para poder prevenir estas enfermedades (RoyoBordonada et al., 2017). El consumo de una alimentación saludable y alta en ácidos grasos insaturados, ha demostrado numerosos beneficios para la salud, como: reducir la prevalencia de síndrome metabólico, diabetes mellitus, ECV (enfermedades cardiovasculares), cáncer mamario y deterioro psicoorgánico (Urquiaga et al., 2017). Los ácidos grasos saturados (AGS) son de síntesis endógena, necesarios para algunas funciones fisiológicas y estructurales. Se ha encontrado una relación positiva entre la ingesta de AGS con el aumento del colesterol total y colesterol LDL (low-density lipoprotein), que a su vez aumenta el riesgo de padecer enfermedad coronaria (CabezasZábala et al., 2016). Asimismo, algunos estudios han demostrado que las concentraciones de colesterol total, LDL, VLDL (very low-density lipoprotein), y TG (triglicéridos) disminuyen al sustituir carne roja por pescado, debido a su alto contenido de grasas monoinsaturadas (Sotos-Prieto et al., 2011). Sustituir AGS por monoinsaturados (AGM) y poliinsaturados (AGP) reduce el riesgo cardiovascular (Ros et al., 2015). Berciano y Ordovás (2014) encontraron que a pesar de que la carne roja puede ser un factor de riesgo para las enfermedades cardiovasculares, esto va más en relación a su contenido y tipo de grasa. La alimentación es una influencia directa sobre las fracciones lipídicas séricas, por lo que se busca el desarrollo de métodos y el diseño de diferentes estrategias nutricionales, para modificar situaciones de hipercolesterolemia e hipertrigliceridemia (Cruz, 2018).

OCDE (2019) refiere que el cerdo ha estado presente mucho tiempo en la historia de la humanidad, pero aún existen prejuicios respecto de su calidad y efectos en la salud. Se considera que el crecimiento de la producción mundial de carne de cerdo será a una tasa promedio anual del $0.9 \%$ entre 2018 y 2027 , para ubicarse en 130.9 millones de toneladas (FIRA, 2019).

Por lo que se siguen buscando estrategias de alimentación para los cerdos, con el afán de bajar precios de producción, así como para mejorar características nutricionales de la carne, vida de anaquel, palatabilidad, entre otros; se ha experimentado con diferentes técnicas de alimentación y uso de fuentes alimenticias convencionales y no convencionales (Montero et al., 2015). El rendimiento y la calidad cárnica, se puede modular incluyendo fuentes ricas en ácidos grasos y antioxidantes. Estas estrategias conducen a mejorar la constitución bioquímica de la carne de consumo. Una de las estrategias para convertir la carne en un "alimento funcional" (Valenzuela et al., 2014), consiste en modificar el perfil de ácidos grasos intramuscular y subcutáneo, con la finalidad de que éstos sean preferentemente insaturados, para mayor aportación hacia la 
salud del consumidor. El hecho de modificar las grasas de la carne del cerdo, coadyuva en los problemas actuales de sobrepeso y obesidad, por lo tanto, previene la incidencia de enfermedades de tipo cardiovascular, diabetes tipo II, hígado graso y síndrome metabólico (Savino, 2011). Una estrategia valorada es la utilización de aguacate en la dieta del cerdo, su uso tuvo un impacto significativo en el contenido y la composición de la grasa intramuscular, reduciendo el contenido de lípidos en los músculos Longissimus thoracis, aumentaron los ácidos grasos poliinsaturados, tocoferol y disminuyó la oxidación de la grasa y proteína (Hernández-López et al., 2016ą; Hernández-López et al., $2016^{b}$ ). Con el objetivo de estudiar el consumo de lomos de cerdos que fueron alimentados con harina de aguacate al $10 \%$, se realizó un estudio para evaluar el efecto en dos periodos de tiempo sobre perfiles antropométricos y de lípidos séricos en personas con sobrepeso.

\section{MATERIAL Y MÉTODOS}

\section{Ubicación}

El presente trabajo fue realizado en el laboratorio de Fisiología Nutricional y Cirugía Experimental de la Unidad Académica de Agricultura, perteneciente a la Universidad Autónoma de Nayarit.

\section{Obtención y características nutricionales de la carne de cerdo}

Se utilizaron 70 kilos de lomos de cerdos de razas comerciales Yorkshire-Landrace, alimentados a libre acceso con una dieta que incluyó harina de aguacate al $10 \%$ (LHA), durante un periodo de 56 días hasta finalizar la engorda, de acuerdo con las recomendaciones de la Norma Oficial Mexicana NOM-062-ZOO-1999. Los aguacates de la variedad Hass (Persea americana Mill) incluidos en la dieta de los cerdos, fueron de descarte comercial por su tamaño, sin demeritar su valor nutricional (Lemus et al., 2017). Los lomos utilizados se caracterizaron en un trabajo anterior (Lemus-Avalos et al., 2020), como un producto cárnico de composición química muy similar, con bajo contenido en grasa intramuscular (5.94\%), con $20.93 \%$ de proteína, $10.98 \mu \mathrm{g} / \mathrm{g}$ de $\mathrm{Y}$-tocoferol, 3128 $\mathrm{mg}$ de ácido gálico/100 $\mathrm{g}$ de fenoles totales, con una alta actividad antioxidante analizado con los ensayos del radical DPPH (2,2- Difenil-1-picrilhidrazilo, D-9132) (477.16 $\mu \mathrm{M}$ Trolox/100g) y del radical ABTS (ácido 2,2'-azino-bis(3-etilbenzotiazolin)-6-sulfónico, A1888) (1054.55 $\mu \mathrm{M}$ Trolox/100 g). Es una carne baja en AGS, incluyendo el palmítico (C16:0) (21.93\%), que es reconocido como la grasa saturada más alta en la carne de cerdo; con mayor cantidad de Araquídico (20:0) (0.45\%). Con un alto contenido de ácidos grasos poliinsaturados, donde el ácido graso esencial Linoleico cis (alfa) (18:2 n6) representa el $12.91 \%$, así como un alto contenido de AGP (13.82\%), por lo que tiene una relación alta de AGP/AGS (0.45 \%) y AGP/AGM (0.25\%).

\section{Sujetos de estudio}

Para el estudio se eligieron 20 participantes adultos masculinos entre las edades de 30 a 60 años, con un IMC (índice de masa corporal) entre 27 a $33 \mathrm{~kg} / \mathrm{m}^{2}$, sin antecedentes de 
enfermedades cardiovasculares, ingesta de carne de cerdo en promedio una vez a la semana, sin realización de actividad física, sin uso de suplementos alimenticios con ácidos grasos omegas, ni medicamentos hipolipemiantes, no fumadores, además de aceptar por escrito participar en el estudio. Esta investigación está apegada a la declaración de Helsinki, a la Ley General de Salud y cuenta con aprobación de la Comisión Estatal de Bioética de Nayarit (CEBIOÉTICA de Nayarit) plasmado en el número de oficio 88/CEB/2018, No. de registro CEBN/17/2018.

Se formaron dos grupos de estudio, ambos consumieron diariamente $100 \mathrm{~g}$ de lomo de cerdo alimentados con harina de aguacate al $10 \%$ (LHA). Un grupo de 10 sujetos (grupo A), lo consumió 4 semanas. El otro (grupo B) integrado con 10 sujetos, lo consumió por un periodo de 6 semanas. La historia nutricional de los individuos se obtuvo por medio de una encuesta directa.

\section{Dieta}

A los participantes se les capacitó para que cumplieran durante el experimento las recomendaciones de una alimentación balanceada de acuerdo a la OMS (OMS, 2017), en base a las características de dieta saludable. La asesoría para el plan de alimentación fue de manera individualizada, de acuerdo al peso, talla y edad de cada sujeto, conforme a la recomendación de la FAO (Carbajal, 2013), se incluyó en su dieta habitual la ingesta del lomo de cerdo proporcionado en el estudio.

\section{Parámetros antropométricos}

Se usó la evaluación antropométrica para determinar la composición corporal y se diagnosticó el grado de obesidad, utilizando el índice de masa corporal (IMC) y la clasificación de obesidad propuesta por la Organización Mundial de la Salud (OMS, 2020). Se realizó la medición antropométrica al inicio y final de cada periodo en ambos grupos, utilizando una báscula marca Tanita® con una sensibilidad de $\pm 100 \mathrm{~g}$, y una báscula digital con bluetooth marca Benetre®; se obtuvo la medida del peso que se realizó en ropa ligera y sin calzado, y por método de bioimpedancia los valores de porcentaje de grasa visceral y porcentaje de grasa corporal. Con la cinta métrica, marca Secaß fijada a una pared lisa se obtuvo la altura $(\mathrm{cm})$, respetando el plano de Frankfurt (porion-infraorbitario) paralelo al suelo y la línea bipupilar horizontal, apoyando la barra horizontal del estadímetro sobre el cuero cabelludo (Martínez y Ortiz, 2013). El IMC se calculó como la razón entre el peso en kilogramos y la altura al cuadrado en metros, considerando el sobrepeso como un IMC igual o superior 25 (OMS, 2017).

Para la obtención del perímetro de cintura $(\mathrm{cm})$ y cadera $(\mathrm{cm})$ se utilizó la cinta métrica metálica Lufkinß, todas las mediciones se realizaron utilizando técnicas internacionalmente aceptadas (Marfell-Jones, M. (ISAK) 2001). Se dividió el perímetro de cintura $(\mathrm{cm})$ entre la cadera $(\mathrm{cm})$ para obtener el indicador cintura/cadera (ICC) como indicador de la distribución del tejido adiposo, este indicador es un predictor independiente de factores de riesgo y morbilidad. Se consideró moderado de 0.90 a 0.95 y alto mayor de 0.95 (Poirier et al., 2006). 


\section{Perfil de lípidos séricos}

En ambos grupos $\mathrm{A}$ y $\mathrm{B}$, se le realizaron muestreos de sangre a los participantes al inicio $y$ al final del periodo de cuatro o seis semanas del consumo de LHA. La muestra se obtuvo de la vena antecubital después de 12 horas de ayuno, se recolectó una muestra basal de sangre de $7 \mathrm{ml}$ en tubo seco. Cada muestra fue colocada en tubos de ensayo y centrifugada a $4.000 \mathrm{rpm}$ por 10 minutos después de la cual fue extraído el suero. Para la determinación de la concentración sérica se midieron con espectrofotómetro Byosistem modelo A160. Obteniendo mediciones de: glucosa $\mathrm{mg} / \mathrm{dL}$, (CT) colesterol total $\mathrm{mg} / \mathrm{dL}$, (TG) triglicéridos $\mathrm{mg} / \mathrm{dL}, \mathrm{TGO}$ (transaminasa glutámica oxalacética) $\mathrm{U} / \mathrm{L}$, colesterol HDL (lipoproteínas de alta densidad) mg/dL, HbA1c (hemoglobina glicada) \%, el colesterol LDL (lipoproteínas de baja densidad) $\mathrm{mg} / \mathrm{dL}$, fue calculado a partir del CT, TG y HDL utilizando la fórmula de Friedewald (LDL=CT-HDL-TG/5) siempre que $\mathrm{TG}<400 \mathrm{mg}$ (Troyo-Barriga, 2004). Para estimar el valor del colesterol VLDL (lipoproteínas de muy baja densidad) $\mathrm{mg} / \mathrm{dL}$, se dividió la concentración de triglicéridos por 5 (mg/dL) (D'lsa et al., 2013).

\section{Análisis estadísticos}

Con los datos antropométricos y los perfiles lipídicos séricos obtenidos, se realizaron análisis descriptivos y comparación entre variables, empleando análisis cuantitativo con $T$ pareada, para comparar en cada grupo las diferencias entre los periodos inicio y final del consumo de LHA. También se realizó en las variables de los perfiles séricos, un análisis cualitativo por medio de la prueba de $\mathrm{X}^{2}$, comparando las proporciones de acuerdo a los niveles $(B)$ bajos, $(N)$ normales $o(A)$ altos en cada grupo comparando las diferencias entre los periodos inicio y final del consumo de LHA. Para el análisis se empleó el software SPSS $\circledast$ Statistics 20 (SSPS, 2011).

\section{RESULTADOS}

El Cuadro 1 presenta las variables antropométricas de los sujetos estudiados, se observa que en los participantes incluidos en ambos grupos ( $A$ y $B$ ) disminuyeron significativamente $(p<0.05)$ la grasa corporal e IMC, observando que cuando se aumenta el periodo a seis semanas de consumo de LHA (grupo B), disminuyen significativamente además la grasa visceral y el perímetro de la cintura.

En el Cuadro 2, se presentan los valores promedio del grupo $A$ y del grupo $B$, de los perfiles lipídicos séricos de los participantes que consumieron LHA. Se muestra que en el grupo A (4 semanas) disminuyeron significativamente $(p<0.05)$ los valores de triglicéridos y VLDL, con aumento de LDL; también se observan que no se afectan la glucosa, colesterol, TGO, HDL y HbA1c. En el grupo B (6 semanas), solo disminuyó significativamente la glucosa $(p<0.05)$; el resto de las variables no fueron afectadas por el consumo LHA. 
Cuadro 1. Antropometría en participantes que consumieron lomos de cerdos alimentados con harina de aguacate al $10 \%$

\begin{tabular}{|c|c|c|c|c|c|c|c|c|}
\hline \multirow[b]{3}{*}{ VARIABLE } & \multicolumn{4}{|c|}{ Grupo A } & \multicolumn{4}{|c|}{ Grupo B } \\
\hline & \multicolumn{2}{|l|}{ Medias } & \multicolumn{2}{|c|}{ Diferencias } & \multicolumn{2}{|c|}{ Medias } & \multicolumn{2}{|c|}{ Diferencias } \\
\hline & I & $\mathrm{F}$ & DIF F-I & $\mathrm{p}<$ & I & $\mathrm{F}$ & DIF F-I & $\mathrm{p}<$ \\
\hline Grasa corporal & 34.76 & 30.72 & -4.04 & ** & 28.9 & 27.6 & -1.28 & ** \\
\hline IMC & 33.04 & 32.12 & -0.92 & ** & 30.9 & 29.6 & -1.26 & ** \\
\hline Grasa visceral & 16.6 & 15.8 & -0.8 & ns & 14.6 & 14.2 & -0.4 & ** \\
\hline Perímetro cintura & 106.7 & 104.2 & -2.5 & ns & 99.8 & 95.4 & -4.4 & ** \\
\hline ICC & 0.98 & 0.96 & -0.02 & ns & 0.92 & 0.90 & -0.02 & ns \\
\hline
\end{tabular}

Grupo A: Consumo por 4 semanas. Grupo B: Consumo por 6 semanas. IMC: Índice de masa corporal, ICC: Índice cintura-cadera. I: inicio de consumo de la carne. F: final de consumo de carne DIF: diferencias, ns: no significativa. ${ }^{* *} p<0.05$ de la prueba T pareada.

Cuadro 2. Perfiles de lípidos séricos en los participantes que consumieron lomos de cerdos alimentados con harina de aguacate al $10 \%$ durante los dos periodos de consumo

\begin{tabular}{|c|c|c|c|c|c|c|c|c|c|}
\hline & & \multicolumn{4}{|c|}{ Grupo A } & \multicolumn{2}{|c|}{ Grupo B } & & \\
\hline \multicolumn{2}{|c|}{ PERIODO DE TIEMPO } & \multirow{2}{*}{$\frac{\text { Media }}{\mathrm{I}}$} & \multicolumn{2}{|c|}{ Diferencias } & \multirow[b]{2}{*}{$\mathrm{p}<$} & \multicolumn{2}{|c|}{ Media } & \multicolumn{2}{|l|}{ Diferencias } \\
\hline & & & $\mathrm{F}$ & $\mathrm{F}-\mathrm{I}$ & & 1 & $\mathrm{~F}$ & $\mathrm{~F}-\mathrm{I}$ & $p<$ \\
\hline GLUCOSA & $\mathrm{mg} / \mathrm{dL}$ & 111.2 & 100.0 & -11.2 & ns & 107.2 & 88.7 & -18.5 & * \\
\hline COLESTEROL & $\mathrm{mg} / \mathrm{dL}$ & 194.6 & 220.2 & 25.6 & ns & 185.8 & 169.1 & -16.7 & ns \\
\hline TRIGLICERIDOS & $\mathrm{mg} / \mathrm{dL}$ & 218.4 & 166.8 & -51.6 & ** & 157.8 & 136.2 & -21.6 & ns \\
\hline TGO & $\mathrm{U} / \mathrm{L}$ & 29.4 & 28 & -1.4 & ns & 31.1 & 28.1 & -3 & ns \\
\hline HDL & $\mathrm{mg} / \mathrm{dL}$ & 58.2 & 52.7 & -5.5 & ns & 52.0 & 50.6 & -1.4 & ns \\
\hline LDL & $\mathrm{mg} / \mathrm{dL}$ & 95.9 & 144.9 & 48.9 & ** & 131.1 & 79.6 & -51.4 & ns \\
\hline VLDL & $\mathrm{mg} / \mathrm{dL}$ & 43.6 & 33.3 & -10.3 & ** & 31.6 & 27.9 & -3.6 & ns \\
\hline $\mathrm{HbA1c}$ & $\%$ & 4.8 & 4.7 & -0.1 & ns & 4.9 & 4.8 & -0.0 & ns \\
\hline
\end{tabular}

Grupo A: Consumo por 4 semanas. Grupo B: Consumo por 6 semanas. I: inicio de consumo de la carne. F: final de consumo de carne. TGO: Aminotransferasa de aspartato, HDL: Lipoproteínas de alta densidad, LDL: Lipoproteínas de baja densidad, VLDL: Lipoproteínas de muy baja densidad, HbA1c: hemoglobina glicada. ${ }^{* *} p<0.05$; ${ }^{*} p<0.10$ de la prueba T pareada.

Se compararon los cambios porcentuales de los perfiles de lípidos séricos de inicio y fin del estudio (cuadro 3), en los participantes del grupo A (4 semanas) se observó que incrementaron significativamente los niveles a valores normales de glucosa (de 40 a $60 \%$ ) y triglicéridos (de 20 a 60\%). El porcentaje de colesterol y LDL se incrementó a rangos altos. En VLDL los porcentajes fueron tendientes a niveles bajos. El resto de variables no sufrieron modificación. Los individuos del grupo B (6 semanas) incrementaron el porcentaje de individuos con valores dentro de los rangos normales de glucosa (de 40 a $90 \%$ ), colesterol (de 80 a 90\%), triglicéridos (de 40 a $70 \%$ ), TGO (de 0 a 10\%), HDL (de 70 a $90 \%$ ) y LDL (de 50 a $90 \%$ ). En los resultados de VLDL incrementó el porcentaje de individuos con valores dentro de los rangos bajos y la $\mathrm{HbA1c}$ no se modificó. Por lo tanto, se muestran mejores resultados para la salud en los participantes del grupo B. 
Cuadro 3. Cambios porcentuales de perfiles de lipídicos sanguíneos en participantes que consumieron lomos de cerdo alimentados con harina de aguacate al $10 \%$

\begin{tabular}{|c|c|c|c|c|c|c|c|c|c|c|c|c|c|c|}
\hline \multicolumn{7}{|c|}{ GRUPO A } & \multicolumn{8}{|c|}{ GRUPOB } \\
\hline VARIABLES & & I & & & $\mathbf{F}$ & & I vs $F$ & & I & & & $\mathbf{F}$ & & I vs F \\
\hline NIVELES & B & $\mathbf{N}$ & A & B & $\mathbf{N}$ & A & $\mathrm{p}<\mathrm{X}^{2}$ & B & $\mathbf{N}$ & A & B & $\mathbf{N}$ & A & $p<x^{2}$ \\
\hline GLUCOSA \% & 0 & 40 & 60 & 0 & 60 & 40 & ** & 0 & 40 & 60 & 0 & 90 & 10 & $* * *$ \\
\hline COLESTEROL\% & 0 & 60 & 40 & 0 & 40 & 60 & $* *$ & 0 & 80 & 20 & 0 & 90 & 10 & ** \\
\hline TRIGLICERIDOS \% & 0 & 20 & 80 & 0 & 60 & 40 & $* * *$ & 0 & 40 & 60 & 0 & 70 & 30 & $* * *$ \\
\hline TGO \% & 80 & 20 & 0 & 80 & 20 & 0 & ns & 100 & 0 & 0 & 90 & 10 & 0 & $* * *$ \\
\hline HDL \% & 0 & 80 & 20 & 0 & 80 & 20 & ns & 10 & 70 & 20 & 0 & 90 & 10 & $* * *$ \\
\hline LDL \% & 20 & 80 & 0 & 0 & 80 & 20 & $* * *$ & 30 & 50 & 20 & 10 & 90 & 0 & $* * *$ \\
\hline VLDL \% & 20 & 40 & 40 & 60 & 40 & 0 & $* * *$ & 40 & 50 & 10 & 70 & 30 & 0 & $* * *$ \\
\hline $\mathrm{HbA} 1 \mathrm{c} \%$ & 0 & 100 & 0 & 0 & 100 & 0 & ns & 0 & 100 & 0 & 0 & 100 & 0 & ns \\
\hline
\end{tabular}

Grupo A: Consumo por 4 semanas. Grupo B: Consumo por 6 semanas. I: Inicio de consumo de la carne. F: Final de consumo de carne de cerdo. Porcentaje de participantes que se encuentran en niveles B: Bajo, N: normal, A: Alto. Glucosa (70-100mg/dL), Colesterol (125-200mg/dL), Triglicéridos (10-150mg/dL), TGO: aminotransferasa de aspartato (40-50U/L), HDL: Lipoproteínas de alta densidad (35-65mg/dL), LDL: Lipoproteínas de baja densidad (13-65 mg/dL), VLDL: Lipoproteínas de muy baja densidad (2-30mg/dL), HbA1c: hemoglobina glicada (4-5,6\%). $\mathrm{p}<$ : Probabilidad estadística de la prueba $\mathrm{X}^{2}\left({ }^{* * *} \mathrm{p}<0.001 ;{ }^{* *} \mathrm{p}<0.05\right.$; ns: No significativa).

\section{DISCUSIÓN}

\section{Parámetros antropométricos}

Este estudio fue diseñado para evaluar el efecto en dos periodos de consumo de LHA sobre los perfiles antropométricos y de lípidos séricos en personas con sobrepeso. Se observó que todos los participantes del estudio disminuyeron significativamente grasa corporal e IMC, mostrando que cuando se aumenta el periodo de consumo de LHA a seis semanas (grupo B), se tuvieron mejores resultados, ya que disminuyeron también las variables grasa visceral y cintura, esto pudiera explicarse por el contenido de ácido linoleico de la carne, considerado ácido graso esencial que beneficia la reducción de grasa corporal (Arenas y López, 2009) y peso corporal (Sanhueza et al., 2002).

Celada et al. (2015) estudiaron a personas con riesgo cardiovascular, añadieron productos cárnicos, donde se sustituyó la grasa animal por una combinación de aceite de oliva, linaza y pescado, en tres periodos de 4 semanas cada uno, reportaron disminución de grasa corporal, relación cintura-cadera y perímetro de cintura en el periodo de alimentación reducida en grasa y se redujo el IMC, mejoró el peso ideal en el periodo de dieta enriquecida con ácidos grasos; estos reportes coinciden con el estudio aquí realizado ya que también disminuyen los parámetros antropométricos en individuos que consumen alimentos funcionales; lo que sugiere que la ingesta de productos cárnicos con menos grasa o enriquecidos con AGI, mejora los marcadores antropométricos pudiendo reducir el riesgo a desarrollar ECV.

Existe una correlación entre IMC y porcentaje de grasa corporal (PGC); de acuerdo a esto, se puede entender la disminución del IMC al bajar el PGC. Así mismo se determinó 
que la prevalencia del tejido adiposo visceral, se relaciona con el IMC y la circunferencia de cadera (García et al., 2016). El incremento del tejido adiposo abdominal (visceral), propicia una mayor síntesis y liberación de adipoquinas además de leptina, resistina, angiotensinógeno, citoquinas pro-inflamatorias (factor de necrosis tumoral [TNF]- $\alpha$, interleuquina [IL]-6, inhibidor del activador del plasminógeno tipo 1 [PAl1]) y quemoquinas, que pueden deteriorar el metabolismo lipídico y glucídico a través del aumento de la resistencia a la insulina, e incrementan el riesgo cardiovascular por lo que medir el índice cintura/cadera (ICC) es útil en la predicción del riesgo cardiometabólico Hernández-Rodríguez et al. (2018).

De acuerdo con González-Acevedo et al. (2013) los cambios pudieran deberse a que la suplementación con AGI inhibe la diferenciación de pre-adipocitos e incrementa la apoptosis de estas células, así como también intervienen en la regulación sobre el sistema nervioso simpático, en la producción de leptina y adiponectina. Dicha función se manifiesta con la regulación de la grasa corporal lo que conlleva a cambios en la distribución de la proporción de masa libre de grasa (Parra y Hernández, 2017).

\section{Perfil de lípidos séricos}

Se observó que el consumo de LHA, en los individuos del grupo A (4 semanas) disminuyó significativamente los niveles TG, VLDL y aumentó LDL. Se muestran también los valores de los individuos del grupo B (6 semanas), observándose diferencias significativas en la disminución de glucosa.

Se observan mejores resultados cuando se consumió por más tiempo (6 semanas) de LHA porque se modificaron mayor número de variables favorables en la salud. El contenido de ácido graso esencial Linoleico cis de la carne consumida se puede asociar a los efectos antiaterogénicos encontrados, por su acción hipocolesterolémica e hipotrigliceridémica (Sanhueza et al., 2002). Así como los compuestos fenólicos de la carne por sus propiedades antioxidantes desempeñan un papel importante en la prevención de enfermedades como desórdenes cardiovasculares, diabetes tipo 2, entre otras (Zapata et al., 2014).

Resultados similares reportaron Petzke et al., (2011), en un estudio realizado en mujeres jóvenes, sanas y de peso normal que consumieron diariamente $200 \mathrm{~g}$ de carne de cerdo magro durante cuatro semanas, observando que disminuyeron significativamente las concentraciones de colesterol total (-7\%), colesterol LDL (-8\%) y glucosa (-4\%). DelgadoPando et al. (2014), realizaron un estudio en 22 voluntarios para evaluar el efecto de consumo de salchichas frankfurters y pate, reducido en grasa y enriquecido con omegas, tras su uso en 3 periodos de 4 semanas, con descansos entre ellos de 4 semanas; encontrando disminución de c-LDL, c-LDL oxidado y cociente c- LDL / c-HDL en los participantes. Resultados similares observaron Maciá et al. (2005), cuando estudiaron personas con hipercolesterolemia (18 mujeres y 18 hombres), consumieron $100 \mathrm{~g} /$ día de jamón Ibérico de Bellota y aceite de oliva, los participantes presentaron un descenso del 
colesterol total, triglicéridos y del colesterol LDL cuando se consumieron tanto AGP como jamón ibérico de bellota, se mantuvieron con niveles normales de HDL.

Los resultados presentados en esta investigación, coinciden con lo observado por Pastoriza et al. (2017), quienes realizaron un estudio con la ingesta de un alimento funcional y observaron que los participantes redujeron los niveles de glucosa en sangre significativamente, debido a las catequinas presentes en la dieta y su acción sobre las enzimas para el control de hiperglucemia post pandrial.

Para TG nuestros resultados coinciden con Díaz-Perilla y Toro (2004) quienes evaluaron una dieta rica en grasa monoinsaturada por 4 semanas que redujo significativamente los triglicéridos en un 18.1\%. Se considera que el efecto es mayor si se parte de hipertrigliceridemia comparado con niveles normales de triglicéridos (Leslie, 2007), mismo efecto que se pudo producir en este trabajo.

En el metaanálisis de (López-Huertas, 2012) se concluye que existe suficiente evidencia para afirmar que la ingesta de dosis > $1 \mathrm{~g}$ de AGI en pacientes con síndrome metabólico durante al menos 3 meses producen una reducción significativa de los triglicéridos plasmáticos en un rango variable de entre $7-25 \%$.

La presencia de compuestos fenólicos, entre ellos los flavonoides, pudiera explicar el efecto hepatoprotector mostrado por el consumo de LHA debido a su actividad antioxidante demostrada, como resultado de una combinación de sus propiedades quelantes de hierro y secuestradoras de radicales libres (Bermúdez-Toledo et al., 2014). Además de los compuestos fenólicos presentes, en los LHA, otros antioxidantes naturales con efectos inmunomoduladores, anti-inflamatorios, antifibróticos, secuestradores de radicales libres y estabilizadores de membrana; pudieran contribuir a proteger al hígado de cambios degenerativos. Así como la presencia del $\gamma$-tocoferol y su metabolito 2,7,8-trimetil-2- ( $\beta$-carboxietil) -6-hidroxicroman ya han demostrado tener actividad antiinflamatoria (Fernandes et al., 2011), por la inhibición de la actividad enzimática de ciclooxigenasa-2 (COX-2), (Muñoz-Velázquez et al., 2012).

Algunos autores consideran que una ingesta dietética equilibrada y adecuada puede desempeñar un papel beneficioso cuando el perfil de lípidos en la sangre es desfavorable y sugieren que el riesgo de dislipidemia puede modificarse según el tipo de grasa ingerida (Peou et al., 2016), se considera que el ácido graso oleico presente en el aguacate, puede desempeñar un papel activo en la reducción de colesterol y triglicéridos en plasma, así como disminuir el c-LDL sin mayor cambio en el c-HDL, reduciendo el riesgo de ECV.

Disminuir el consumo de fuentes nutricionales con AGS ayuda a prevenir el desarrollo de padecer diabetes mellitus tipo 2 (Mirmiran et al., 2018). Un estudio de revisión publicado en el 2017 reportó que los sujetos que tienen una dieta baja en AGS mostraron mejores perfiles antiinflamatorios, o al menos una respuesta proinflamatoria (Rocha et al., 2017). Estos hallazgos sugieren que el consumo de alimentos con AGI (ácidos grasos 
insaturados), contribuye a mejorar el estado inflamatorio y en consecuencia prevenir el desarrollo de resistencia a la insulina, diabetes y enfermedades cardiovasculares.

Se considera en este estudio que el tratamiento alimentario estuvo modificado con respecto a los ácidos grasos de igual modo que en otros estudios, (Jiya et al., 2015). El perfil de ácidos grasos en los animales monogástricos es casi un reflejo directo de los ácidos grasos de su alimentación; por lo tanto, los LHA que se consumieron por los participantes contienen AGS preferentemente modificados por AGI logrando mejorar el perfil lipídico (Lemus-Avalos et al., 2020). Al emplear carne de cerdo alimentado con aguacate, no solo se incorpora a la carne para consumo humano AGP sino también, antioxidantes que pueden beneficiar la salud al consumidor. (Hernández-López et al., 2016ạ; Hernández-López et al., 2016b).

\section{CONCLUSIÓN}

La ingesta diaria de lomo de cerdo alimentado con harina de aguacate al $10 \%$, en los participantes de los dos períodos de consumo de 4 y 6 semanas disminuyeron significativamente los parámetros antropométricos grasa corporal e IMC, asociados con sobrepeso, alteraciones metabólicas y cardiovasculares. En los perfiles de lípidos séricos disminuyeron triglicéridos, VLDL y glucosa; se incrementaron los porcentajes a rangos normales de glucosa, colesterol y triglicéridos, con mejor resultado en el grupo B con 6 semanas de consumo de lomo de cerdo alimentado con harina de aguacate al $10 \%$.

\section{LITERATURA CITADA}

ARENAS AM, López MSI. 2009. Efecto de las dietas enriquecidas con ácido linoleico conjugado (CLA) sobre la composición lipídica del músculo e hígado y la composición química de la lubina (Dicentrarchus labrax). Vector plus: miscelánea científico-cultural. (33):61-70.

https://accedacris.ulpgc.es/bitstream/10553/6400/1/0231633_00033_0004.pdf

BERCIANO S, Ordovás JM. 2014. Nutrición y salud cardiovascular. Revista española de cardiología. 67(9):738-747. https://doi.org/10.1016/j.recesp.2014.05.003

BERMÚDEZ-TOLEDO D, Escobar-Román R, Boffill-Cárdenas M, Betancourt-Morgado E, Igualada-Correa I, Alonso-Cáceres B. 2014. Evaluación del potencial hepatoprotector de la Mentha piperita $L$ previo a la inducción de hepatotoxicidad con acetaminofen. Boletín latinoamericano y del Caribe de plantas medicinales y aromáticas. 13(6):545-556. ISSN: 0717-7917. https://www.redalyc.org/pdf/856/85632545005.pdf

CABEZAS-ZÁBALA CC, Hernández-Torres BC, Vargas-Zárate M. 2016. Aceites y grasas: efectos en la salud y regulación mundial. Revista de la Facultad de Medicina. 64(4): 761-768. ISSN: 0120-0011.

http://www.scielo.org.co/pdf/rfmun/v64n4/0120-0011-rfmun-64-04-00761.pdf 
CARBAJAL AA. 2013. Manual de Nutrición y Dietética. Departamento de Nutrición. Facultad de Farmacia. Universidad Complutense de Madrid. Madrid, España. Pp. 6-350. https://eprints.ucm.es/22755/1/Manual-nutricion-dietetica-CARBAJAL.pdf

CELADA P, Delgado-Pando G, Olmedilla-Alonso B, Jiménez-Colmenero F, Ruperto M, Sánchez-Muniz FJ. 2015. Impact of improved fat-meat products consumption on anthropometric markers and nutrient intakes of male volunteers at increased cardiovascular risk. Nutrición hospitalaria. 32(2): 710-721. ISSN: 0212-1611. https://www.redalyc.org/pdf/3092/309243317031.pdf

CRUZ GY. 2018. Sobre las asociaciones entre los lípidos séricos y el riesgo cardiovascular. Revista Cubana de Alimentación y Nutrición. 28(1):125-151. RNPS: 2221. ISSN: 1561-2929. https://www.medigraphic.com/pdfs/revcubalnut/can2018/can181k.pdf

DELGADO-PANDO G, Celada P, Sánchez-Muniz FJ, Jiménez-Colmenero F, OlmedillaAlonso B. 2014. Effects of improved fat content of frankfurters and pâtés on lipid and lipoprotein profile of volunteers at increased cardiovascular risk: a placebo-controlled study. European journal of nutrition. ISSN: 1436-6207.53(1): 83-93. https://link.springer.com/article/10.1007/s00394-013-0502-1

DIAZ-PERILLA M, Toro CA. 2004. Efecto de la adición de aguacate a la alimentación habitual sobre los niveles de lípidos en personas con dislipidemia. Universitas Scientiarum. 9(2):49-58.

ISSN:

0122-7483.

https://www.redalyc.org/pdf/499/49990206.pdf

D’ISA BG, Chilelli C, Areny G, Zacharzewski C. 2013. Colesterol-LDL ¿determinación química, fórmula de Friedewald o análisis de Regresión? Medicina Infantil. 20(3):234238. http://www.medicinainfantil.org.ar/images/stories/volumen/2013/xx_3_234.pdf

FERNANDES Â, Barreira J, Barros L, Antonio AL, Bento A, Botelho ML, Ferreira IC. 2011. Efeito protector da radiação y nos níveis de vitamina $\mathrm{E}$ em Castanea sativa. $V$ Jornadas de Análises Clínicas e Saúde Pública de Bragança. Portugal 20 e 21 de Maio de 2011. http://hdl.handle.net/10198/9521 https://bibliotecadigital.ipb.pt/handle/10198/9521

FIRA (Fidecomisos instituidos en relación con la agricultura). 2019. Panorama Agroalimentario / Carne de cerdo 2018. Dirección de investigación y evaluación económica y sectorial. México. https://www.fira.gob.mx/Nd/index.jsp\#

GARCÍA A, Niño-Silva L, González-Ruiz K, Ramírez-Vélez R. 2016. Volumen de grasa visceral como indicador de obesidad en hombres adultos. Revista Colombiana de Cardiología. 23(4):313-320. ISSN 0120-5633.

http://dx.doi.org/10.1016/j.rccar.2015.12.009

http://www.scielo.org.co/pdf/rcca/v23n4/v23n4a15.pdf 
GONZÁLEZ-ACEVEDO O, Hernández-Sierra JF, Salazar-Martínez A, Mandeville PB, Valadez-Castillo FJ, de la Cruz-Mendoza E. 2013. Efecto de la suplementación de omega 3 sobre IMC, ICC y composición corporal en mujeres obesas. Archivos Latinoamericanos de Nutrición. 63(3):224. ISSN 0004-0622.

https://www.alanrevista.org/ediciones/2013/3/art-5/

HERNÁNDEZ-RODRÍGUEZ J, Moncada Espinal OM, Domínguez YA. 2018. Utilidad del índice cintura/cadera en la detección del riesgo cardiometabólico en individuos sobrepesos y obesos. Revista Cubana de Endocrinología. 29(2): 1-16. ISSN: 1561-2953. http://scielo.sld.cu/pdf/end/v29n2/end07218.pdf

HERNÁNDEZ-LÓPEZ SH, Rodríguez-Carpena JH, Lemus-Flores C, Grageola-Nuñez F, Estévez M. 2016a. Avocado waste for finishing pigs: Impact on muscle composition and oxidative stability during chilled storage. Meat Science. ISSN: 0309-1740. 116: 186-192. http://doi.org/10.1016/j.meatsci.2016.02.018

HERNÁNDEZ-LÓPEZ SH, Rodríguez-Carpena JG, Lemus-Flores C, Galindo-García J, Estévez M. 2016 ${ }^{\mathrm{b}}$. Antioxidant protection of proteins and lipids in processed pork loin chops through feed supplementation with avocado. Journal of Food Science and Technology. 53(6). 2788-2796. ISSN:1365-2621. http://doi.org/10.1007/s13197-0162252-6

JIYA EZ, ljaiya AT, Ayanwale BA, Olorunsanya AO. 2015. Fatty acid composition of meat from the hind leg cut of rabbits (Oryctolagus cunniculus) fed diets containing graded levels of processed tallow (Detarium microcarpum) seed meal. Biotechnology in Animal Husbandry. 31(2):283-290. ISSN: 1450-9156. https://doi.org/10.2298/BAH1502283J

LEMUS-AVALOS G, Lemus-Flores C, Bugarín-Prado JO, Grageola-Núñez F, AyalaValdovinos MA, Duifhuis-Rivera T, Moo-Huchin VM, Dzib-Cauich D. 2020. Effect of diets with avocado meal on lipids in muscle, antioxidants and gene expression in finished pigs. Revista Bio Ciencias. 7:e968. ISSN 2007-3380. https://doi.org/10.15741/revbio.07.e968

LEMUS C, Bugarín J, Grageola F, Rodríguez JG, Mejía K, Valdivia R. 2017. Características químicas de la pasta de aguacate hass fruto completo (Persea americana Mill.) Mexicano de Nayarit destinado a la alimentación animal. Revista Computadorizada de Producción Porcina. 24(2). 212-218. ISSN: 1026-9053. http://www.iip.co.cu/RCPP/242/06\%20CLemus.pdf

LESLIE WS, Hankey CR, Lean ME. 2007. Weight gain as an adverse effect of some commonly prescribed drugs: a systematic review. QJM: An International Journal of Medicine. 100(7):395-404. https://academic.oup.com/qjmed/article/100/7/395/2948323 
LÓPEZ-HUERTAS E. 2012. The effect of EPA and DHA on metabolic syndrome patients: a systematic review of randomised controlled trials. British journal of nutrition. 107(S2):S185-S194. ISSN: 0007-1145. https://www.cambridge.org/core/journals/britishjournal-of-nutrition/article/effect-of-epa-and-dha-on-metabolic-syndrome-patients-a-

systematic-review-of-randomised-controlledtrials/C7371DEB03026DF9B3CFE637DB1E3901

LEY GENERAL DE SALUD. Reglamento de la ley general de salud en materia de investigación para la salud. México; 1987.

http://www.salud.gob.mx/unidades/cdi/nom/compi/rlgsmis.html

MACIÁ BE, Ortiz-Cansado A, García-Rebollo AJ, García-Domínguez M, Morales-Blanco P, Benito-Hernández J. 2005. Dieta rica en ácido oléico procedente de jamón lbérico de Bellota frente a una dieta rica en ácidos grasos poliinsaturados omega-3 y omega-6. Efecto sobre el perfil lipídico en una población hipercolesterolémica. Suppl 1. Nutr. Hosp. 135-136. ISSN: 0212-1611. http://scielo.isciii.es/pdf/nh/v20s1/area8.pdf

MARFELL-JONES M. 2001. Manual ISAK: estándares de medidas antropométricas internacionales. Sociedad Internacional para el Avance de la Cineantropometría: ISBN 0 868037125 5 712 República de Sudáfrica. Pp ix-xxii. https://antrofor.files.wordpress.com/2020/02/manual-isak-2005-cineantropometriacastellano1.pdf

MARTÍNEZ SJM, Ortiz-Moncada R. 2013. Antropometría: manual básico para estudios de salud pública, nutrición comunitaria y epidemiología nutricional. Departamentos, Enfermería, Enf. comunitaria, Medicina Preventiva y salud pública e historia de la ciencia. Facultad Ciencias de la Salud, Universidad de Alicante, España. http://rua.ua.es/dspace/handle/10045/28100 https://rua.ua.es/dspace/bitstream/10045/28100/1/Martinez_y_Ortiz_ANTROPOMETRIA manual_basico_SP_NC_y_Epi_2013.pdf

MIRMIRAN P, Esfandyari S, Moghadam SK, Bahadoran Z, Azizi F. 2018. Fatty acid quality and quantity of diet and risk of type 2 diabetes in adults: Tehran Lipid and Glucose Study. Journal of Diabetes and its Complications. 32(7):655-659. ISSN: 1056-8727. https://doi.org/10.1016/j.jdiacomp.2018.05.003

https://www.sciencedirect.com /science/article/abs/pii/S1056872718300035

MONTERO LEM, Martínez GRG, Herradora LMA. 2015. Alternativas para la producción porcina a pequeña escala. ISBN: 978-607-02-6915-8 http://www.fmvz.unam.mx/fmvz/publicaciones/archivos/Alternativas_Porcina.pdf 
MUÑOZ-VELÁZQUEZ EE, Rivas-Díaz K, Loarca-Piña M, Flavia G, Mendoza-Díaz S, Reynoso-Camacho R, Ramos-Gómez M. 2012. Comparación del contenido fenólico, capacidad antioxidante y actividad antiinflamatoria de infusiones herbales comerciales. Revista mexicana de ciencias agrícolas. 3(3): 481-495. ISSN: 2007-0934. http://www.scielo.org.mx/pdf/remexca/v3n3/v3n3a6.pdf

NOM (Norma Oficial Mexicana, NOM-062-ZOO-1999) de especificaciones técnicas para la producción, cuidado y uso de los animales de laboratorio. México. http://cbsuami.org/documentos/labsdivisionales/bioterio/NOM-062-ZOO.pdf

OCDE (Organización para la Cooperación y el Desarrollo Económicos). 2019. Exámenes de mercado en México: estudio de caso del mercado de la carne de cerdo. https://www.oecd.org/daf/competition/market-examinations-mexico-pork-meat-marketweb-esp.pdf

OMS (Organización mundial de la salud (OMS). 2017. Boletín de Enfermedades cardiovasculares. https://www.who.int/es/news-room/fact-sheets/detail/cardiovasculardiseases-(cvds)

OMS (Organización mundial de la salud (OMS). 2020. Obesidad y sobrepeso https://www.who.int/es/news-room/fact-sheets/detail/obesity-and-overweight

PARRA A, Hernández S. 2017. Enfermedades cardiovasculares y nutrición. En KauferHorwitz M, Pérez-Lizaur AB, Arroyo P. Nutriología médica. México: Médica Panamericana. 4ta. Edición. Pp. 594-619. ISBN 9786079356415 https://www.medicapanamericana.com/mx/libro/nutriologia-medica

PASTORIZA S, Mesías M, Cabrera C, Rufian-Henares JA. 2017. Healthy properties of green and white teas: an update. Food \& function. 8(8): 2650-2662. ISSN: 20426496. https://pubs.rsc.org/en/content/articlelanding/2017/fo/c7fo00611j/unauth\#!divAbstract

PETZKE KJ, Lemke S, Klaus S. 2011. Increased fat-free body mass and no adverse effects on blood lipid concentrations 4 weeks after additional meat consumption in comparison with an exclusion of meat in the diet of young healthy women. Journal of nutrition and metabolism. 2011. PMID: 21773015. ISSN: 20900732. https://www.ncbi.nlm.nih.gov/pmc/articles/PMC3136131/

PEOU S, Milliard-Hasting B, Shah SA. 2016. Impact of avocado-enriched diets on plasma lipoproteins: A meta-analysis. Journal of clinical lipidology. 10(1): 161-171. ISSN: 1933-2874. https://doi.org/10.1016/j.jacl.2015.10.011 https://www.sciencedirect.com/science/article/pii/S1933287415004274 
POIRIER P, Giles TD, Bray GA, Hong Y, Stern JS, Pi-Sunyer FX, Eckel RH. 2006. Obesity and cardiovascular disease: pathophysiology, evaluation, and effect of weight loss: an update of the 1997 American Heart Association Scientific Statement on Obesity and Heart Disease from the Obesity Committee of the Council on Nutrition, Physical Activity, and Metabolism. Circulation. 113(6): 898-918. ISSN:

1524-4539.

https://doi.org/10.1161/CIRCULATIONAHA.106.171016 https://www.ahajournals.org/doi/full/10.1161/CIRCULATIONAHA.106.171016

ROCHA DM, Bressan J, Hermsdorff HH. 2017. The role of dietary fatty acid intake in inflammatory gene expression: a critical review. Sao Paulo Medical Journal. 135(2): 157168. ISSN: 1516-3180. http://dx.doi.org/10.1590/1516-3180.2016.008607072016 https://www.scielo.br/scielo.php?pid=S1516-31802017000200157\&script=sci_arttext

ROS E, López-Miranda J, Picó C, Rubio MÁ, Babio N, Sala-Vila A, Gil HA. 2015. Consenso sobre las grasas y aceites en la alimentación de la población española adulta: postura de la Federación Española de Sociedades de Alimentación, Nutrición y Dietética (FESNAD). Nutrición Hospitalaria. 32(2): 435-477. ISSN: 0212-1611. http://dx.doi.org/10.3305/nh.2015.32.2.9202

http://scielo.isciii.es/scielo.php?script=sci_arttext\&pid=S0212-16112015000800001

ROYO-BORDONADA M, Lobos J, Brotons C, Villar F, de Pablo C, Armario P. 2017. El estado de la prevención cardiovascular en España. Dialnet. 142 (1):7- 14. ISSN 00257753 https://dialnet.unirioja.es/servlet/articulo?codigo=4533331

SANHUEZA J, Nieto S, Valenzuela A. 2002. Ácido linoleico conjugado: un ácido graso con isomería trans potencialmente beneficioso. Revista chilena de nutrición. 29(2): 98105. ISSN: 0717-7518. http://dx.doi.org/10.4067/S0717-75182002000200004 https://scielo.conicyt.cl/scielo.php?script=sci_arttext\&pid=S0717-75182002000200004

SAVINO P. 2011. Obesidad y enfermedades no transmisibles relacionadas con la nutrición. Revista colombiana de cirugía. 26(3):180-195. ISSN: 2011-7582 https://www.redalyc.org/pdf/3555/355535509008.pdf

SOTOS-PRIETO M, Guillen M, Sorlí JV, Asensio E, Gillem Sáiz P, González JI, Corella D. 2011. Consumo de carne y pescado en población mediterránea española de edad avanzada y alto riesgo cardiovascular. Nutrición Hospitalaria. 26(5):1033-1040. ISSN: 0212-1611. http://scielo.isciii.es/pdf/nh/v26n5/17_original_16.pdf

SPSS. 2011. Statistical Package for the Social Sciences, IBM® SPSS® Statistics 20. for Windows: advanced statistic release. SPSS, Chicago Illinois. http://www.spss.com.hk/corpinfo/history.htm 
TROYO-BARRIGA P. 2004. Obesidad y dislipidemias. Gaceta Medica México. 140(2): 49-58. https://www.medigraphic.com/pdfs/gaceta/gm-2004/gms042g.pdf

URQUIAGA I, Echeverría G, Dussaillant C, Rigotti A. 2017. Origen, componentes y posibles mecanismos de acción de la dieta mediterránea. Revista médica de Chile. 145(1): 85-95. ISSN: 0034-9887. http://dx.doi.org/10.4067/S0034-98872017000100012

VALENZUELA R, Morales I, González A, Morales P, Sanhueza C. Valenzuela B. 2014. Ácidos grasos poliinsaturados de cadena larga $\omega-3$ y enfermedad cardiovascular. Revista Chilena de Nutrición. 41(3):319-327. ISSN: 0716-1549. www.redalyc.org/pdf/469/46932089014.pdf

WOOD JD, Enser M. 2017. Chapter 20 - Manipulating the fatty acid composition of meat to improve nutritional value and meat quality. In New Aspects of Meat Quality. Pp. 501 535. Woodhead Publishing. España. ISBN: 9780081005934. https://doi.org/10.1016/B978-0-08-100593-4.00023-0 https://www.sciencedirect.com/science/article/pii/B9780081005934000230

WORLD MEDICAL ASSOCIATION. World Medical Association Declaration of Helsinki: ethical principles for medical research involving human subjects. 2013. Jama 310(20): 2191-2194. http://dx.doi.org/10.1001/jama.2013.281053 https://jamanetwork.com/journals/jama/article-abstract/1760318

ZAPATA S, Piedrahita AM, Rojano B. 2014. Capacidad atrapadora de radicales oxígeno (ORAC) y fenoles totales de frutas y hortalizas de Colombia. Perspectivas En Nutrición Humana. 16(1):25-36. https://revistas.udea.edu.co/index.php/nutricion/article/view/20310 